\title{
DUST OBSCURATION IN STARBURST GALAXIES FROM NEAR-INFRARED SPECTROSCOPY
}

\author{
DANiela Calzettit ${ }^{1}$ and ANNE L. KinNey ${ }^{1}$ \\ Space Telescope Science Institute, 3700 San Martin Drive, Baltimore, MD 21218 \\ AND \\ THAISA STORCHI-BERGMANN \\ Instituto de Fisica, Universidad Federal Rio Grande do Sul, Porto Alegre, Brazil \\ Received 1995 June 5; accepted 1995 August 11
}

\begin{abstract}
We present new observations of the near-infrared hydrogen recombination lines $\mathrm{Pa} \beta$ and $\mathrm{Br} \gamma$ in the central starburst regions of 13 galaxies. The near-infrared data are supplemented with data on the optical Balmer line emission and are used to study the geometrical distribution of the dust associated with the massive stars. We find that the reddening toward the starburst regions can be explained by the presence of foreground dust, either homogeneous or clumpy. The result suggests that there is little internal dust in the star-forming site. Mechanisms like shock waves and outflows produced by supernova explosions and hot star winds can be effective at destroying and removing the dust from inside the region.
\end{abstract}

Subject headings: dust, extinction — galaxies: ISM — galaxies: starburst — infrared: galaxies

\section{INTRODUCTION}

The study and the interpretation of the physical and chemical properties of external galaxies depend on how effectively we can take into account the effects of dust obscuration. In those extragalactic objects in which nebular emission is present (e.g., star-forming galaxies), the effect of dust opacity is usually removed from the spectra by attributing to dust obscuration any difference between the observed and the intrinsic ratio of hydrogen emission lines. Three assumptions are implicit in this process: (1) case $B$ recombination is applicable to the intrinsic $\mathrm{H}$ line intensity; (2) the Galactic extinction curve, which is usually adopted, is applicable to the extragalactic object; and (3) the dust in the object is distributed in a foreground screen.

The first two assumptions are reasonably valid in most cases. Case B recombination is probably a good approximation of the physical conditions of the gas for many galaxies. The two known extragalactic extinction curves, the Large Magellanic Cloud and the Small Magellanic Cloud curves, are similar in shape to the Galactic extinction curve at optical, and probably also longer, wavelengths (see, however, Calzetti, Kinney, \& Storchi-Bergmann 1994). The assumption that the dust is spatially distributed in a foreground screen is more problematic. Intuition suggests that complex geometries should be expected for the dust distribution: the dust can be mixed with the gas in the interstellar space (internal dust) and can be clumped. Scattering of photons by dust grains into the line of sight can contribute to the "blueing" of an otherwise reddened spectrum (Bruzual, Magris, \& Calvet 1988; Witt, Thronson, \& Capuano 1992).

The spatial distribution of the dust associated with the massive stars in a burst of star formation can be investigated via the $\mathrm{H}$ line ratios at different wavelengths. For instance, near-infrared (NIR) emission lines suffer lower extinction, and therefore probe larger optical depths, than optical lines. A few studies of $\mathrm{H}$ emission lines in starburst galaxies found that the NIR extinction is in general larger than the optical extinction,

\footnotetext{
${ }^{1}$ Visiting Astronomer, Kitt Peak National Observatory, operated by the Association of Universities for Research in Astronomy, Inc. under contract with the National Science Foundation.
}

which suggests that the dust may be clumped or heavily mixed with the emitting gas (Moorwood \& Oliva 1988; Kawara, Nishida, \& Phillips 1989; Puxley 1991; Puxley \& Brand 1994). However, given the intrinsic weakness of the NIR H lines relative to the optical $\mathrm{H}$ lines and the small differential extinction between infrared lines, even a small measurement uncertainty translates into a large uncertainty in the final extinction value.

Starburst environments may be inhospitable for dust: the large rate of supernova explosions and the hot star winds produce shock waves that may enhance the destruction rate of dust (Draine \& Salpeter 1979a, b) and may generate large-scale outflows of gas and dust (Heckman, Armus, \& Miley 1990).

Calzetti et al. (1994) showed that the ultraviolet and optical spectra of starburst galaxies have reddening characteristics compatible with a dust screen in front of the bursting region. For the same galaxies, about $70 \%$ of the IRAS far-infrared dust emission is compatible with foreground dust heated by massive stars (Calzetti et al. 1995; Meurer et al. 1995).

Whether the "foreground screen" scenario (either homogeneous or clumpy) is generally applicable to starburst regions has not been proved yet. For this purpose, we have obtained $J$ and $K$ spectra of the starburst central regions of a sample of 13 galaxies and measured the $\mathrm{Pa} \beta$ and $\mathrm{Br} \gamma$ emission lines. By supplementing the infrared data with measurements of the Balmer emission lines from existing optical spectra, we study the geometrical distribution of dust in the sample galaxies.

\section{OBSERVATIONS}

The infrared cryogenic spectrometer (CRSP) with the 300 line $\mathrm{mm}^{-1}$ grating at the KPNO $1.3 \mathrm{~m}$ telescope was employed to obtain $J$ and $K$ spectra of the 13 galaxies listed in Table 1 during two separate runs in 1994 April and September. The galaxies are part of a larger sample for which we have optical spectra, from 3200 to $8000 \AA$ (Calzetti et al. 1994; Kinney et al. 1994; Storchi-Bergmann, Calzetti, \& Kinney 1994; McQuade, Calzetti, \& Kinney 1995; Storchi-Bergmann, Kinney, \& Challis 1995). The optical spectra were obtained in a circular aperture of $13^{\prime \prime} .5$ diameter or in a $10^{\prime \prime} \times 20^{\prime \prime}$ rectangular aperture (NGC 4385, NGC 1614, and NGC 7714). In order to compare the properties of the optical and the infrared emis- 
TABLE 1

Observational Data and Derived Quantities

\begin{tabular}{|c|c|c|c|c|c|c|c|c|}
\hline \multirow[b]{2}{*}{ GalaXY } & \multirow[b]{2}{*}{$z^{\mathrm{a}}$} & \multirow[b]{2}{*}{$E(B-V)_{G}^{b}$} & \multirow{2}{*}{$\begin{array}{c}\operatorname{Pa} \beta(1.28 \mu \mathrm{m}) \\
\left(\mathrm{ergs} \mathrm{s}^{-1} \mathrm{~cm}^{-2}\right)\end{array}$} & \multirow{2}{*}{$\begin{array}{c}\operatorname{Br} \gamma(2.17 \mu \mathrm{m}) \\
\left(\mathrm{ergs} \mathrm{s}^{-1} \mathrm{~cm}^{-2}\right)\end{array}$} & \multicolumn{4}{|c|}{$E(B-V)$} \\
\hline & & & & & $\mathrm{H} \alpha / \mathrm{H} \beta$ & $\mathrm{Pa} \beta / \mathrm{Br} \gamma$ & $\mathrm{H} \beta / \mathrm{Br} \gamma$ & $\mathrm{H} \gamma / \mathrm{Pa} \beta$ \\
\hline IC $1586 \ldots$ & 0.020 & 0.02 & $1.65 \pm 0.12(-14)$ & $0.52 \pm 0.11(-14)$ & $0.57 \pm 0.09$ & $1.38 \pm 0.55$ & $0.41 \pm 0.08$ & $0.29 \pm 0.12$ \\
\hline IC $214 \ldots \ldots$ & 0.032 & 0.03 & $4.25 \pm 0.30(-14)$ & $0.91 \pm 0.08(-14)$ & $0.53 \pm 0.11$ & $0.45 \pm 0.28$ & $0.70 \pm 0.05$ & $0.69 \pm 0.15$ \\
\hline NGC $1569 \ldots \ldots$ & 0.000 & 0.51 & $33.13 \pm 1.65(-14)$ & $7.16 \pm 0.36(-14)$ & $0.06 \pm 0.07$ & $-0.01 \pm 0.17$ & $0.13 \pm 0.04$ & $0.13 \pm 0.07$ \\
\hline NGC $1614 \ldots \ldots$ & 0.016 & 0.05 & $22.31 \pm 0.45(-14)$ & $7.90 \pm 0.71(-14)$ & $0.93 \pm 0.06$ & $1.63 \pm 0.22$ & $0.97 \pm 0.04$ & $0.87 \pm 0.04$ \\
\hline NGC $4194 \ldots \ldots$ & 0.008 & 0.00 & $19.51 \pm 0.59(-14)$ & $5.25 \pm 0.26(-14)$ & $0.80 \pm 0.08$ & $1.02 \pm 0.15$ & $0.65 \pm 0.04$ & $0.62 \pm 0.05$ \\
\hline NGC $4385 \ldots \ldots$ & 0.007 & 0.02 & $6.60 \pm 0.33(-14)$ & $2.47 \pm 0.32(-14)$ & $0.60 \pm 0.07$ & $1.79 \pm 0.33$ & $0.52 \pm 0.05$ & $0.34 \pm 0.06$ \\
\hline NGC $4861 \ldots \ldots$ & 0.003 & 0.00 & $15.57 \pm 0.31(-14)$ & $3.05 \pm 0.18(-14)$ & $0.20 \pm 0.06$ & $0.27 \pm 0.16$ & $0.20 \pm 0.04$ & $\ldots$ \\
\hline NGC $5860 \ldots \ldots$ & 0.017 & 0.01 & $2.35 \pm 0.24(-14)$ & $0.77 \pm 0.20(-14)$ & $0.69 \pm 0.17$ & $1.48 \pm 0.66$ & $0.61 \pm 0.10$ & \\
\hline NGC $6052 \ldots \ldots$ & 0.015 & 0.03 & $3.97 \pm 0.28(-14)$ & $1.17 \pm 0.18(-14)$ & $0.20 \pm 0.08$ & $1.21 \pm 0.40$ & $0.29 \pm 0.06$ & $0.15 \pm 0.06$ \\
\hline NGC $6090 .$. & 0.029 & 0.00 & $8.17 \pm 0.25(-14)$ & $2.51 \pm 0.15(-14)$ & $0.59 \pm 0.06$ & $1.34 \pm 0.17$ & $0.60 \pm 0.04$ & $0.49 \pm 0.04$ \\
\hline NGC $7250 \ldots \ldots$ & 0.004 & 0.15 & $4.58 \pm 0.37(-14)$ & $0.97 \pm 0.12(-14)$ & $0.09 \pm 0.07$ & $0.31 \pm 0.35$ & $0.23 \pm 0.05$ & $0.20 \pm 0.05$ \\
\hline NGC $7673 \ldots \ldots$ & 0.010 & 0.04 & $5.37 \pm 0.32(-14)$ & $1.13 \pm 0.19(-14)$ & $0.41 \pm 0.11$ & $0.40 \pm 0.43$ & $0.40 \pm 0.07$ & $0.39 \pm 0.06$ \\
\hline NGC $7714 \ldots \ldots$ & 0.009 & 0.04 & $19.30 \pm 0.58(-14)$ & $4.99 \pm 0.20(-14)$ & $0.39 \pm 0.06$ & $0.89 \pm 0.13$ & $0.41 \pm 0.03$ & $0.34 \pm 0.04$ \\
\hline
\end{tabular}

${ }^{a}$ Redshift.

${ }^{\mathrm{b}}$ Foreground Galactic color excess.

sion, we matched closely the infrared aperture to the optical one. The CRSP slit was opened to 6".9, and a window $20^{\prime \prime}$ wide was extracted from the frames. The resolution is $R \sim 450$ in both bands. The CRSP long slit covers $146^{\prime \prime}$ on the sky along the spatial axis, and the orientation was set on the east-west direction during both observing runs.

Multiple exposures of 3 and 5 minutes at $J$ and $K$, respectively, were obtained for each object to account for the rapid fluctuations of the sky background at infrared wavelengths. On-target exposures were alternated by sky frames in order to subtract the $\mathrm{OH}$ and $\mathrm{O}_{2}$ emission lines. Each galaxy observation was bracketed by the observation of a $G$ dwarf star at less than 0.1 air mass from the target to remove telluric absorption lines from the final spectra. On-target total exposure times were typically $18-25$ minutes in $J$ and $25-30$ minutes in $K$.

The data were background subtracted and flat-fielded using standard IRAF packages. Argon and krypton lamps were used for the wavelength calibration. The wavelength range covered by the spectra is $1.236-1.340 \mu \mathrm{m}$ in $J$ and $2.113-2.250 \mu \mathrm{m}$ in $K$.

Two to three photometric standard stars of spectral type A from the list of Elias et al. (1982) were observed each night for the absolute flux calibration. We assumed that the Elias et al. standards approximate blackbody spectral distributions of appropriate temperatures in order to use photometric data to flux-calibrate spectra. Hydrogen absorption lines were removed from all the stellar spectra in order to avoid the introduction of artificial features in the calibrated galaxy spectra. The calibration of the stellar fluxes shows a variation by only a few percent from night to night during the same observing run and by less than $5 \%$ in $J$ and less than $3 \%$ in $K$ between the April and the September runs. The photometric uncertainties in the galaxy spectra range from $7 \%$ to $20 \%$, due in some cases to nonperfect atmospheric conditions.

\section{ANALYSIS AND RESULTS}

The fluxes and $1 \sigma$ uncertainties of the $\mathrm{Pa} \beta$ and the $\mathrm{Br} \gamma$ lines for the 13 galaxies are given in Table 1. Two of the galaxies in our sample (NGC 1614 and NGC 7714) have been previously studied in the near-infrared by other authors. We compare our $\mathrm{H}$ recombination line measurements with theirs to check for photometric accuracy. The aperture size employed for the different observations is critical, since the emitting region in star- burst galaxies is extended. Our Bry measurements of NGC 1614 and NGC 7714 are in agreement within the uncertainties with measurements reported by authors who used comparable aperture sizes (Ho, Beck, \& Turner 1990; Kawara et al. 1989; Aitken, Roche, \& Phillips 1981). Measurements of $\mathrm{Pa} \beta$ for the two galaxies are reported by Puxley \& Brand (1994), and their values are about $20 \%$ and $50 \%$ lower than ours for NGC 1614 and NGC 7714, respectively; we attribute the difference to the fact that they employ an aperture $3^{\prime \prime} .1 \times 9$ ".3, much smaller than the one we use.

The photometry of our optical spectra is typically accurate to $20 \%-25 \%$ (see McQuade et al. 1995; Storchi-Bergmann et al. 1995). The mismatch between the optical and NIR line fluxes due to differences in the aperture sizes should be small, since our apertures are large enough to encompass most of the line-emitting regions (Calzetti et al. 1995). For instance, NGC 1614 and NGC 7714 were observed with both the circular and the rectangular optical apertures; line measurements from the two sets of spectra gave consistent results to better than $10 \%$, despite the difference of about $40 \%$ between the areas of the two apertures. In summary, photometric uncertainties between optical and NIR line fluxes should be no larger than $\sim 30 \%$.

Ratios from $\mathrm{H}$ recombination lines at different wavelengths are used to study the geometrical distribution of the dust associated with the massive stars in the starburst regions. Independent values of the color excess $E(B-V)$ are derived from the different $\mathrm{H}$ line ratios, according to the standard formula:

$$
E(B-V)_{H_{a} / H_{b}}=\frac{\log \left(R_{\mathrm{obs}} / R_{\mathrm{int}}\right)}{0.4\left[k\left(\lambda_{a}\right)-k\left(\lambda_{b}\right)\right]},
$$

where $H_{a}$ and $H_{b}$ are two $\mathrm{H}$ emission lines with intrinsic ratio $R_{\text {int }}$ and observed ratio $R_{\text {obs }}$, and $k(\lambda)$ is the extinction curve. Equation (1) provides the actual value of the color excess in the observed spectra only if (1) the dust is foreground and homogeneously distributed and (2) the extinction curve $k(\lambda)$ is appropriate for the case. If both assumptions are satisfied, any $\mathrm{H}$ line ratio gives the same value of the color excess $E(B-V)$. Deviations from this behavior can be attributed to a different dust distribution and/or, possibly, to a different extinction curve.

The $\mathrm{H} \alpha / \mathrm{H} \beta$ ratio is commonly employed to derive color excess values for extragalactic objects because both lines fall in the optical window and are usually intense and easy to 
measure. The $\mathrm{H} \alpha / \mathrm{H} \beta$ ratio is used here as a reference value for comparison with other line ratios. The $\mathrm{Pa} \beta / \mathrm{Br} \gamma$ ratio uses the least obscured emission lines and probes a larger optical depth in the galaxies than the optical lines. However, even a small uncertainty in the ratio translates into a large uncertainty in the extinction value because the extinction curve has small values in the infrared region. The $\mathrm{H} \beta / \mathrm{Br} \gamma$ ratio uses the longest wavelength baseline available with two well-detected lines. A long baseline reduces the effects of the measurement uncertainties (see below). The $\mathrm{H} \gamma$ and $\mathrm{Pa} \beta$ lines have a common upper level, so their ratio is independent of the physical conditions of the gas; however, the $\mathrm{H} \gamma$ line is weak and often noisy in our spectra.

Prior to analysis, the Balmer line intensities are corrected for the underlying absorption due to stars (Calzetti et al. 1994). The underlying stellar absorption is small in the infrared and can be neglected. The adopted intrinsic ratios are $\mathrm{H} \alpha / \mathrm{H} \beta=2.90, \mathrm{~Pa} \beta / \mathrm{Br} \gamma=5.72, \mathrm{H} \beta / \mathrm{Br} \gamma=33.14$, and $\mathrm{H} \gamma /$ $\operatorname{Pa} \beta=2.68$, extrapolating values for a gas with electron temperature $T_{e} \simeq 7500 \mathrm{~K}$ and density $n_{e}=100 \mathrm{~cm}^{-3}$ from Osterbrock (1989). We adopt the Seaton (1979) extinction curve in the visible and the Landini et al. (1984) extinction curve in the infrared.

Table 1 lists the $E(B-V)$ values for all the galaxies. The error bars take into account uncertainties both in the line measurements and in the intrinsic ratios due to electron temperature and density variations $\left(5000 \mathrm{~K} \leq T_{e} \leq 10,000 \mathrm{~K}\right.$, and $10^{2} \mathrm{~cm}^{-3} \leq n_{e} \leq 10^{4} \mathrm{~cm}^{-3}$ ).

The color excesses derived from $\mathrm{H} \beta / \mathrm{Br} \gamma$ and $\mathrm{Pa} \beta / \mathrm{Br} \gamma$ for the 13 starburst regions are shown as a function of $E(B-V)$ from $\mathbf{H} \alpha / \mathbf{H} \beta$ in Figures 1 and 2, respectively. To investigate the effects of different distributions of dust and gas, we consider three simple models (Calzetti et al. 1994): (1) a foreground homogeneous screen; (2) a foreground clumpy screen; and (3) a homogeneous mix of dust and emitting gas (internal dust). In the first model, all the ionized gas is behind a homogeneous

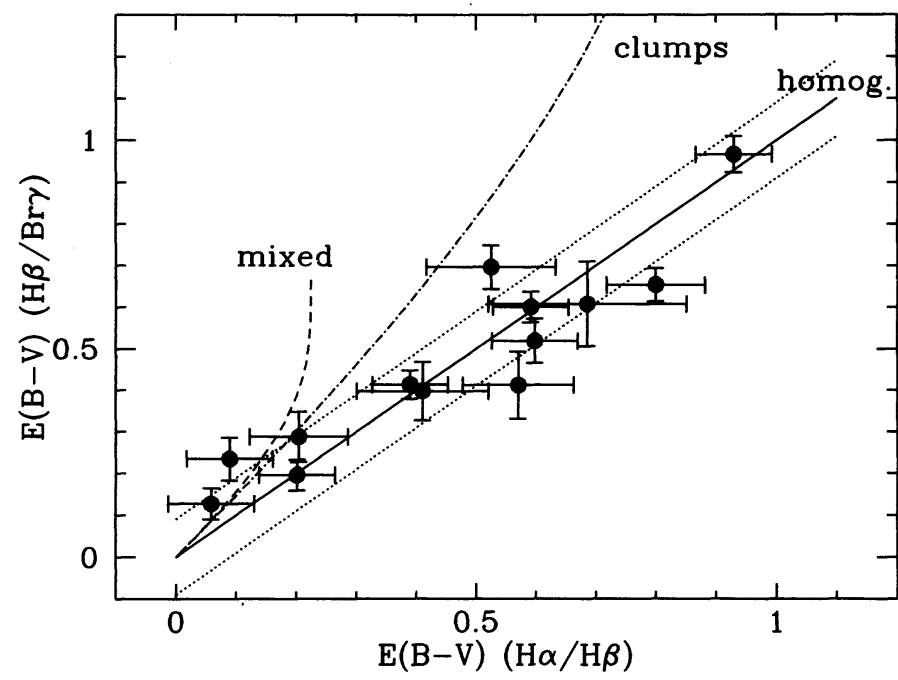

FIG. 1.-The color excess $E(B-V)$ derived from the hydrogen line ratio $\mathrm{H} \beta / \mathrm{Br} \gamma$ is shown as a function of the color excess from the $\mathrm{H} \alpha / \mathrm{H} \beta$ line ratio. The error bars are $1 \sigma$ uncertainties. The data are compared with the following models for the dust distribution: a foreground homogeneous dust screen (continuous line), a clumpy foreground screen (dash-dotted line), and a homogeneous mixture of dust and gas (internal dust, dashed line). The dotted lines mark the range of $\mathrm{H} \beta / \mathrm{Br} \gamma$ values around the screen model expected for a photometric mismatch up to $\sim 30 \%$ between the optical and NIR data.

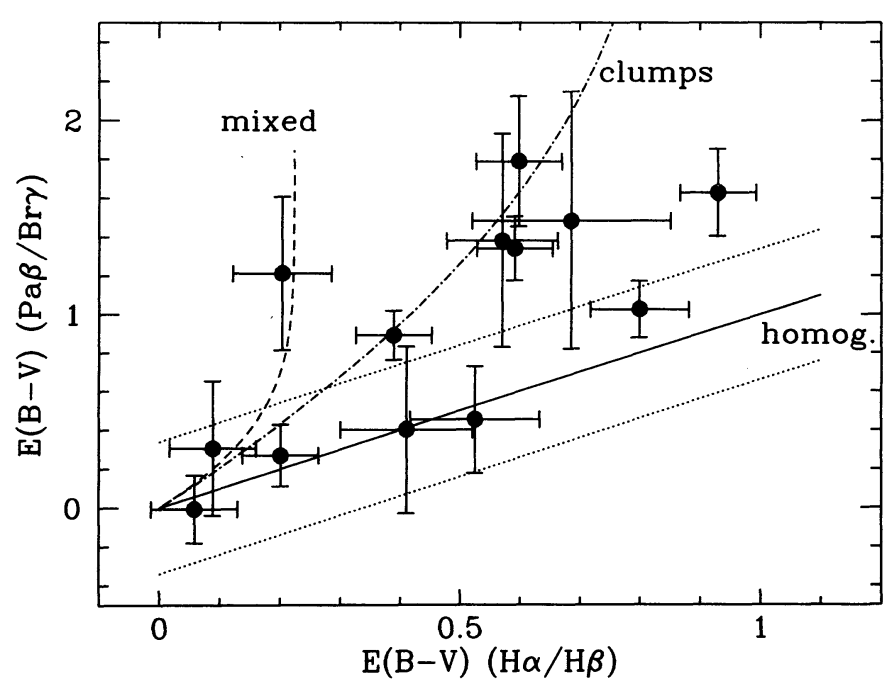

FIG. 2.-Same as Fig. 1, for the line ratio $\mathrm{Pa} \beta / \mathrm{Br} \gamma$. The dotted lines mark the range of $\mathrm{Pa} \beta / \mathrm{Br} \gamma$ values around the screen model expected for an uncertainty $\sim 15 \%$ between the $J$ - and $K$-band fluxes.

slab of dust, and the obscuration depends only on the details of the extinction curve; in a color excess versus color excess plot, a foreground homogeneous screen is represented by a straight line. The slope of the line is unity if the Galactic extinction curve applies to the galaxies. In the second model, the dust is still between the gas and the observer but is distributed in clumps; lines of sight with the lowest number of clumps provide the largest contribution to the integrated emission, and this effect becomes more important going from the infrared to the optical wavelengths. In our model, the clumps are assumed to have all the same optical depth and to be Poissonian-distributed along the line of sight, with average number $\mathcal{N}$ (Natta \& Panagia 1984). In Figures 1 and 2, the lines corresponding to $\mathscr{N}=10$ are drawn; already for $\mathscr{N} \geq$ 20 , the clumpy distribution is almost indistinguishable from a homogeneous distribution (see Calzetti et al. 1994). In the internal dust model, the dust and the gas are homogeneously mixed across the emitting region. External gas layers are less affected by reddening and dimming than internal layers, since they are not as deeply embedded in dust; consequently, they provide most of the contribution to the emerging radiation. Mixed regions appear almost unreddened, though dimmer than a dust-free region. This effect is more pronounced at optical wavelengths, where the dust extinction is more efficient, than at NIR wavelengths, and the color excess calculated using optical emission lines soon reaches asymptotic values (see Figs. 1 and 2). For the second and third models, we determine the color excess versus color excess trends using the Galactic extinction curve and including the effects of scattering; for the albedo and phase function, we use the values given by Natta $\&$ Panagia (1984) in the optical range and the predictions by Kim, Martin, \& Hendry (1994) in the NIR range (A. N. Witt 1995, private communication).

The data in Figure 1 show a better agreement with the foreground homogeneous screen model than with the other two configurations. The same trend is shown by the $\mathrm{Pa} \beta / \mathrm{H} \gamma$ line ratio, though with a larger scatter due to the uncertainties in the $\mathrm{H} \gamma$ line measurements. In Figure 2, the $\mathrm{Pa} \beta / \mathrm{Br} \gamma$ ratios are located between the homogeneous and the clumpy screen models. Figures 1 and 2 appear inconsistent; however, much of 
the disagreement may be due to uncertainties in the photometry of the different wavebands. An uncertainty of $\sim 15 \%$ between the $J$ - and $K$-band fluxes gives a large uncertainty in the color excess, $\delta E(B-V)_{\mathrm{Pa} \beta / \mathrm{Br} \gamma}= \pm 0.34$, because of the small differential values of the NIR extinction curve. For the $\mathrm{H} \beta / \mathrm{Br} \gamma$ ratio, the long-wavelength baseline helps to reduce the effects of the uncertainties: a photometric mismatch as large as $30 \%$ between the optical and NIR spectra produces a small offset in the color excess, $\delta E(B-V)_{\mathrm{H} \beta / \mathrm{Br} \gamma}=0.09$. We show in Figures 1 and 2 how the position of the homogeneous screen model changes as an effect of the photometric uncertainties (dotted lines).

The clear result from our analysis is that the data are not compatible with the case of internal dust alone. Puxley \& Brand (1994) obtained a similar result analyzing the NIR spectra of NGC 1614 and NGC 7714. In particular, they concluded that the dust distribution in NGC 7714 is well described by a homogeneous dust screen, while for NGC 1614 a patchy foreground distribution must be invoked. This is consistent with what we find for the same galaxies (see Table 1).

\section{DISCUSSION AND CONCLUSIONS}

For the 13 galaxies in our sample the reddening toward the massive stars in the starburst regions can be explained by Galactic-type foreground dust, either homogeneous or clumpy. Calzetti et al. $(1994,1995)$ reached similar conclusions from the characteristics of the ultraviolet stellar continuum emission and the far-infrared dust emission. In particular, they showed that about $70 \%$ of the IRAS far-infrared dust emission can be attributed to foreground dust heated by the starburst's massive stars. From these results, we infer that most of the reddeningproducing dust is located outside and surrounding the starburst region.

Starburst regions are characterized by intense shock waves from supernova explosions and from hot star winds. Shocks from supernovae are most likely responsible for the destruction of the dust grains, through grain-grain collisions and sputtering in the warm interstellar medium (Draine \& Salpeter 1979a, b; Draine 1990; Jones et al. 1994). From the IRAS far-infrared luminosity, we derive supernova rates in the range $0.02-0.5$ supernovae per year for our galaxies (Heckman et al. 1990).
Adopting $M=5 \times 10^{7} M_{\odot}$ for the mass of the gas associated with the burst, a typical supernova energy of $10^{51}$ ergs, a median value for the supernova rate of 0.08 supernovae per year, and an effective supernova rate of one-third the measured value (McKee 1989), the average lifetime for a refractory grain is $\sim 10^{6} \mathrm{yr}$. This crude calculation assumes that the burst of star formation is an isolated system and that the ISM in the region has the same structure as the ISM in the Galaxy. However, the grain lifetime we obtain is still at least one order of magnitude smaller than the lifetime of the burst, $t_{\mathrm{SB}} \sim 10^{7} \mathrm{yr}$ for a star formation rate of $\sim 5 M_{\odot} \mathrm{yr}^{-1}$.

Supernova explosions and hot star winds also generate mass outflows, which can develop into large-scale winds ("superwinds"; Heckman et al. 1990) and eject significant amounts of the interstellar gas and dust (De Young \& Heckman 1994). In addition, superwinds may interfere with the dust formation processes, e.g., the grain growth that occurs in the ISM (McKee 1989).

The presence of residual dust inside the regions is not completely excluded by our data. For instance, the emission-line ratios of NGC 1614 are compatible with either a foreground clumpy screen or a composite scenario of foreground homogeneous screen plus internal dust (see Puxley \& Brand 1994). The optical depths at $V$ are $\tau_{V} \sim 2.4$ for the homogeneous screen and $\tau_{V} \sim 3$ for the internal dust. Calzetti et al. (1995) find that $\sim 30 \%$ of the stars heating the dust in starburst galaxies are not detected at UV and optical wavelengths; those stars may be buried deep inside thick dust clouds, so that they are manifest only through the dust emission in the far-infrared. Investigations at longer wavelengths are needed to understand fully the actual amount and the physical conditions of the dust still present inside the starburst regions (e.g., Kawara et al. 1989; Puxley \& Brand 1994).

The authors thank T. M. Heckman, G. Meurer, and M. Voit for many discussions and useful suggestions; A. N. Witt and D. R. Garnett for a critical reading of the manuscript; and R. R. Joyce for the helpful advice during the observation and the reduction of the infrared spectra. D. C. acknowledges support from the NASA grant NAGW-3757 during this research.

\section{REFERENCES}

Aitken, D. K., Roche, P. F. \& Phillips, M. M. 1981, MNRAS, 196, 101P Bruzual A., G., Magris, G. C., \& Calvet, N. 1988, ApJ, 333, 673

Calzetti, D., Bohlin, R. C., Kinney, A. L., Storchi-Bergmann, T., \& Heckman, T. M. 1995, ApJ, 443, 136

Calzetti, D., Kinney, A. L., \& Storchi-Bergmann, T. 1994, ApJ, 429, 582

De Young, D. S., \& Heckman, T. M. 1994, ApJ, 431, 598

Draine, B. T. 1990, in ASP Conf. Proc., 12, The Evolution of the Interstellar Medium, ed. L. Blitz (San Francisco: ÂSP), 193

Draine, B. T., \& Salpeter, E. E. 1979a, ApJ, 231, 77

. 1979b, ApJ, 231, 438

Elias, J. H., Frogel, J. A., Matthews, K., \& Neugebauer, G. 1982, AJ, 87, 1029

Heckman, T. M., Armus, L., \& Miley, G. 1990, ApJS, 74, 833

Ho, P. T. P., Beck, S. C., \& Turner, J. L. 1990, ApJ, 349, 57

Jones, A. P., Tielens, A. G. G. M., Hollenbach, D. J., \& McKee, C. F. 1994, ApJ, 433,797

Kawara, K., Nishida, M., \& Phillips, M. M. 1989, ApJ, 337, 230

Kim, S.-H., Martin, P. G., \& Hendry, P. D. 1994, ApJ, 422, 164

Kinney, A. L., Calzetti, D., Bica, E., \& Storchi-Bergmann, T. 1994, ApJ, 429, 172
Landini, M., Natta, A., Oliva, E., Salinari, P., \& Moorwood, A. F. M. 1984 A\&A, 134, 284

McKee, C. F. 1989, in IAU Symp. 135, Interstellar Dust, ed. L. J. Allamandola \& A. G. G. M. Tielens (Dordrecht: Kluwer), 431

McQuade, K., Calzetti, D., \& Kinney, A. L. 1995, ApJS, 97, 331

Meurer, G. R., Heckman, T. M., Leitherer, C., Kinney, A. L., Robert, C., \& Garnett, D. R. 1995, AJ, submitted

Moorwood, A. F. M., \& Oliva, E. 1988, A\&A, 203, 278

Natta, A., \& Panagia, N. 1984, ApJ, 287, 228

Osterbrock, D. E. 1989, Astrophysics of Gaseous Nebulae (Mill Valley: Univ. Science Books

Puxley, P. J. 1991, MNRAS, 249, 11 P

Puxley, P. J., \& Brand, P. W. J. L. 1994, MNRAS, 266, 431

Seaton, M. J. 1979, MNRAS, 187, 73P

Storchi-Bergmann, T., Calzetti, D., \& Kinney, A. L. 1994, ApJ, 429, 572

Storchi-Bergmann, T., Kinney, A. L., \& Challis, P. 1995, ApJS, 98, 103

Witt, A. N., Thronson, H. A., \& Capuano, J. M. 1992, ApJ, 393, 611 